\title{
On thermal stability of topological qubit in Kitaev's 4D model
}

\author{
R. Alicki ${ }^{1,2}$, M. Horodecki ${ }^{1,2}$, P. Horodecki ${ }^{1,3}$, and R. Horodecki ${ }^{1,2}$ \\ 1 National Quantum Information Centre of Gdańsk, Poland \\ 2 Institute of Theoretical Physics and Astrophysics, University of Gdańsk, Poland \\ ${ }^{3}$ Faculty of Applied Physics and Mathematics, Gdansk University of Technology, Poland
}

\begin{abstract}
We analyse stability of the four-dimensional Kitaev model - a candidate for scalable quantum memory - in finite temperature within the weak coupling Markovian limit. It is shown that, below a critical temperature, certain topological qubit observables $X$ and $Z$ possess relaxation times exponentially long in the size of the system. Their construction involves polynomial in system's size algorithm which uses as an input the results of measurements performed on all individual spins. We also discuss the drawbacks of such candidate for quantum memory and mention the implications of the stability of qubit for statistical mechanics.
\end{abstract}

\section{INTRODUCTION}

While quantum computation offers algorithms which can outperform the classical ones, they are very fragile with respect to external disturbance. Therefore, along with the discoveries of fast algorithms, the question of how to protect quantum computation against decoherence was the subject of extensive studies. As a result the whole domain was created known as fault tolerant quantum computation [1]. The famous threshold theorems $[2,3]$, saying that arbitrary long quantum computation is possible provided the error per gate is below certain threshold has given the hope, that it is possible in principle to overcome the decoherence. However the initial theorems are based on phenomenological model of noise, and the problem, has not been solved within Hamiltonian dynamics [4-7]. Even the problem of whether one can store qubits is open.

There is, though a class of candidates for quantum memories, which are in between realistic description and the phenomenological one: the Kitaev models of topological quantum memory [8-10]. There is a heuristic reasoning, according to which such memories are instable in two dimensions $[8,11]$, and stable in four dimensions (similarly like Ising model represent a stable classical memory in 2D, but not in 1D) [8]. Behaviour of of Kitaev models in finite temperature was then investigated (see e.g. [12-16]). Quite recently the thermal instability of $2 \mathrm{D}$ model has been rigorously proved in [17]. In the present paper, we deal with the 4D Kitaev's model of Ref. [8] and prove rigorously, within Markovian weak coupling approximation, that the model provides thermally stable qubit. To this end we use the formalism of quantum semigroup theory [18], which has been successfully applied to analysis of Kitaev 2D model in Ref. [17]. As a byproduct we obtain a very useful general upper bound for decay rate. We perform our analysis in parallel for $3 \mathrm{D}$ and $4 \mathrm{D}$ case. Indeed, though in $3 \mathrm{D}$ case only one of the qubit observables is stable, as argued in [8], it is much more transparent and the reasoning is the same as in $4 \mathrm{D}$ case. Since the very stability of qubit is not sufficient for a good quantum memory, we also discuss the open problems concerning existence of self-correcting quantum memory. Implications for description of thermodynamical limit are also discussed.

The paper is organized as follows. In section II we provide some basic notions and results concerning Markovian weak coupling limit. We show, in particular, how the rate of decay expressed in terms of noise generator is related to fidelity criterion. Finally we provide a general upper bound for decay rate. In section III we show that analysis of noisy evolution of some particular topological observables is reduced to the study of a classical model. Next (sec. IV) we provide conditions for stability of these observables in terms of one-step autocorrelation functions. In sec. $\mathrm{V}$ we finally prove the stability of the observables. In sec. VI we provide polynomial algorithm to measure the observables. Finally (sec. VII) we discuss remaining open problems for existence of self-correcting quantum memory, as well as importance of the result for description of systems in thermodynamical limit.

\section{MARKOVIAN APPROXIMATION IN WEAK COUPLING LIMIT}

Let us first we briefly sketch the general setup and properties of Davies generators. A quantum system with discrete energy spectrum is coupled to a collection of heat baths leading to the global Hamiltonian

$H=H^{\text {sys }}+H^{\text {bath }}+H^{\text {int }} \quad$ with $\quad H^{\text {int }}=\sum_{\alpha} S_{\alpha} \otimes f_{\alpha}$,

where the $S_{\alpha}$ are system operators and the $f_{\alpha}$ bath operators. The main ingredients are the Fourier transforms $\hat{h}_{\alpha}$ of the autocorrelation functions of the $f_{\alpha}$. The function $\hat{h}_{\alpha}$ describes the rate at which the coupling is able to transfer an energy $\omega$ from the bath to the system. Often a minimal coupling to the bath is chosen, minimal in the sense that the interaction part of the Hamiltonian is as simple as possible but still addresses all energy levels of the system Hamiltonian in order to produce finally an ergodic reduced dynamics. The necessary and sufficient condition for ergodicity is $[19,20]$

$$
\left\{S_{\alpha}, H^{\mathrm{sys}}\right\}^{\prime}=\mathbb{C} \mathbb{1},
$$


i.e. no system operator apart from the multiples of the identity commutes with all the $S_{\alpha}$ and $H^{\text {sys }}$.

We begin by introducing the Fourier decompositions of the $S_{\alpha}$ 's as they evolve in time under the system evolution

$$
\mathrm{e}^{i t H^{\mathrm{sys}}} S_{\alpha} \mathrm{e}^{-i t H^{\mathrm{sys}}}=\sum_{\omega} S_{\alpha}(\omega) \mathrm{e}^{-i \omega t} .
$$

Here the $\omega$ are the Bohr frequencies of the system Hamiltonian. From self-adjointness we have the relation

$$
S_{\alpha}(-\omega)=S_{\alpha}(\omega)^{\dagger}
$$

The weak coupling limit procedure then returns the following equation for the evolution of the spin system in Heisenberg picture

$$
\begin{aligned}
\frac{d X}{d t} & =i\left[H^{\mathrm{sys}}, X\right]+\mathcal{L}_{\mathrm{dis}}(X)=: \mathcal{L}(X) \\
\mathcal{L}_{\mathrm{dis}}(X) & =\frac{1}{2} \sum_{\alpha} \sum_{\omega} \hat{h}_{\alpha}(\omega)\left(S_{\alpha}^{\dagger}(\omega)\left[X, S_{\alpha}(\omega)\right]+\right. \\
& \left.+\left[S_{\alpha}^{\dagger}(\omega), X\right] S_{\alpha}(\omega)\right)
\end{aligned}
$$

For thermal baths one has moreover the relation

$$
\hat{h}_{\alpha}(-\omega)=\mathrm{e}^{-\beta \omega} \hat{h}_{\alpha}(\omega)
$$

which is a consequence of the KMS condition [18]. The operator $\mathcal{L}$ generates a semigroup of completely positive identity preserving transformations of the spin system. It describes the reduced dynamics in the Markovian approximation and enjoys the following properties

- The canonical Gibbs state with density matrix

$$
\rho_{\beta}=\frac{\mathrm{e}^{-\beta H^{\mathrm{sys}}}}{\operatorname{Tr}\left(\mathrm{e}^{-\beta H^{\mathrm{sys}}}\right)}
$$

is a stationary state for the semigroup, i.e.

$$
\operatorname{Tr}\left(\rho_{\beta} \mathrm{e}^{t \mathcal{L}}(X)\right)=\operatorname{Tr}\left(\rho_{\beta} X\right)
$$

- The semigroup is relaxing, i.e. for any initial state $\rho$ of the system

$$
\lim _{t \rightarrow \infty} \operatorname{Tr}\left(\rho \mathrm{e}^{t \mathcal{L}}(X)\right)=\operatorname{Tr}\left(\rho_{\beta} X\right)
$$

- Furthermore, the generator satisfies the detailed balance condition, often called reversibility. Writ$\operatorname{ing} \delta(X):=\left[H^{\text {sys }}, X\right]$,

$$
\left[\delta, \mathcal{L}_{\text {dis }}\right]=0
$$

and

$\operatorname{Tr}\left(\rho_{\beta} Y^{\dagger} \mathcal{L}_{\text {dis }}(X)\right)=\operatorname{Tr}\left(\rho_{\beta}\left(\mathcal{L}_{\text {dis }}(Y)\right)^{\dagger} X\right)$.

The last equation expresses the self-adjointness of the generator with respect to the scalar product defined by the equilibrium state. The space of observables equipped with the scalar product

$$
\langle X, Y\rangle_{\beta}:=\operatorname{Tr} \rho_{\beta} X^{\dagger} Y
$$

is called the Liouville space and the generator of the reduced dynamics is a normal matrix on that space, i.e. the Hermitian and skew-Hermitian parts of the generator commute.

Finally it is known that $-\mathcal{L}$ is a positive operator, hence it has nonnegative eigenvalues. Moreover $L(I)=0$, and for ergodic systems eigenvalue 0 is nondegenerate.

\section{A. Autocorrelation functions, decay rate and fidelity}

Suppose that for observable $X$ satisfying $\langle X, X\rangle_{\beta}=1$ $\langle X, I\rangle_{\beta}=0$ we have

$$
-\langle X, \mathcal{L}(X)\rangle_{\beta} \leq \epsilon
$$

Then the autocorrelation function of the observable satisfies

$$
\left\langle X, e^{\mathcal{L} t} X\right\rangle_{\beta} \geq e^{-\epsilon t}
$$

One proves it easily, by decomposing $X$ into normalized eigenvectors of $\mathcal{L}$, and using convexity of function $e^{-x}$. Thus to show that an observable $X$ is stable, it is enough to estimate $-\langle X, \mathcal{L}(X)\rangle$, which can be therefore called decay rate for the observable $X$. If this quantity decreases exponentially with size of the system, we obtain stability.

Let us now rephrase it in the language of fidelity. Namely, suppose we have observables $X$ and $Z$ satisfy commutation rules of Pauli algebra. They generate subalgebra which defines a virtual qubit, the one to be protected. Let the induced tensor product on the total Hilbert space be

$$
\mathcal{H}=\mathcal{H}_{Q} \otimes \mathcal{H}_{\text {anc }}
$$

Now, we fix some state $\rho_{\text {anc }}$ on the system $\mathcal{H}_{\text {anc }}$. For any state $\psi$ of qubit the initial state of the total system is $\rho_{Q, a n c}(0)=|\psi\rangle\langle\psi| \otimes \rho_{a n c}$. Then the system evolves into state $\rho_{Q \text {,anc }}(t)$, and finally, the ancilla is traced out. Thus the fidelity is given by

$$
F(\psi)=\left\langle\psi\left|\rho_{Q}^{\text {out }}(t)\right| \psi\right\rangle
$$

where

$$
\rho_{Q}^{o u t}(t)=\operatorname{Tr}_{a n c}\left(\rho_{Q, a n c}(t)\right)
$$

Let us denote the fidelity averaged uniformly over the states of qubit by $\bar{F}$.

Proposition 1 With the above notation, suppose now that the Gibbs state is of the form

$$
\rho_{\beta}=\frac{1}{2} \mathrm{I}_{Q} \otimes \rho_{a n c}
$$


where $\rho_{\text {anc }}$ is a state on ancilla. We then have

$$
\bar{F} \geq \frac{1}{2}\left(\left\langle X, e^{\mathcal{L} t} X\right\rangle_{\beta}+\left\langle Z, e^{\mathcal{L} t} Z\right\rangle_{\beta}\right) \geq e^{-\epsilon t}
$$

where $\epsilon$ is upper bound for the rates $-\langle X, \mathcal{L}(X)\rangle_{\beta}$ and $-\langle Z, \mathcal{L}(Z)\rangle_{\beta}$.

Proof. Let $F_{x}$ be given by

$$
F_{x}=\frac{1}{2}(F(|0\rangle)+F(|1\rangle))
$$

where $|0\rangle,|1\rangle$ are eigenstates of $X$ treated as observable on system $Q$. Similarly we define $F_{z}$. Using results of [21] and [22] one finds that

$$
\bar{F} \geq F_{x}+F_{z}-1
$$

Thus it is enough to estimate e.g. $F_{x}$. Using the property (20) and orthogonality $X \perp I$ one finds that

$$
F_{x}=\frac{1}{2}\left(1+\left\langle X, e^{\mathcal{L} t} X\right\rangle_{\beta}\right) .
$$

Combining the last two formulas with (16) ends the proof.

\section{B. Upper bound for decay rate}

We now present a useful bound for decay rate, which holds for operators $X$ which are eigenvectors of $[H, \cdot]$. For such operators, one computes

$$
\begin{aligned}
& -\langle X, \mathcal{L}(X)\rangle_{\beta}=\sum_{\omega \geq 0} \hat{h}(\omega)\left(\left\langle\left[S_{\alpha}(\omega), X\right],\left[S_{\alpha}(\omega), X\right]\right\rangle_{\beta}\right. \\
& \left.+e^{-\omega \beta}\left\langle\left[S_{\alpha}(-\omega), X\right],\left[S_{\alpha}(-\omega), X\right]\right\rangle_{\beta}\right) \leq \\
& \leq 2 \sum_{\omega} \hat{h}(\omega)\left\langle\left[S_{\alpha}(\omega), X\right],\left[S_{\alpha}(\omega), X\right]\right\rangle_{\beta} \leq \\
& \leq 2 \hat{h}_{\max } \sum_{\omega}\left\langle\left[S_{\alpha}(\omega), X\right],\left[S_{\alpha}(\omega), X\right]\right\rangle_{\beta}
\end{aligned}
$$

where

$$
\hat{h}_{\max }=\sup _{\omega \geq 0} \hat{h}(\omega) .
$$

Since $X$ and $S_{\alpha}(\omega)$ are eigenvectors of $[H, \cdot \cdot$ it follows that $\left[S_{\alpha}(\omega), X\right]$ are eigenvectors of $[H, \cdot]$ too, hence they are mutually orthogonal. We thus can write

$\sum_{\omega}\left\langle\left[S_{\alpha}(\omega), X\right],\left[S_{\alpha}(\omega), X\right]\right\rangle_{\beta}=\sum_{\omega, \omega^{\prime}}\left\langle\left[S_{\alpha}(\omega), X\right],\left[S_{\alpha}\left(\omega^{\prime}\right), X\right]\right\rangle_{\beta}$.

However from definition of $S_{\alpha}(\omega)$ it follows that

$$
\sum_{\omega} S_{\alpha}(\omega)=S_{\alpha}
$$

This gives

$$
-\langle X, \mathcal{L}(X)\rangle_{\beta} \leq 2 \hat{h}_{\max } \sum_{\alpha}\left\langle\left[S_{\alpha}, X\right],\left[S_{\alpha}, X\right]\right\rangle_{\beta}
$$

The advantage of the formula is that the only place where the self-Hamiltonian appears is the Gibbs state in scalar product.

\section{FROM QUANTUM TO CLASSICAL IN KITAEV-TYPE MODELS}

We consider a system of $N$ spin- $1 / 2$ systems. For any set $S$ of spins let us denote $X_{S}=\Pi_{j \in S} \sigma_{j}^{x}, Z_{S}=\Pi_{j \in S} \sigma_{j}^{z}$. Consider now Hamiltonian of the form

$$
H=-\sum_{s} X_{s}-\sum_{c} Z_{c}
$$

and we assume that the sets $s$ and the sets $c$ are chosen in such a way that the operators $X_{s}$ and $Z_{c}$ commute with each other. Consider also the following coupling to environment

$$
H_{i n t}=\sum_{j} \sigma_{j}^{x} \otimes f_{j}+\sum_{j} \sigma_{j}^{z} \otimes \tilde{f}_{j} .
$$

Then Davies operators fall into two types:

$$
\begin{aligned}
& a_{\alpha}=\sigma_{x}^{j} P_{\alpha} \\
& b_{\alpha}=\sigma_{z}^{j} R_{\alpha}
\end{aligned}
$$

where $P_{\alpha}$ belong to algebra spanned by those operators $Z_{c}$ whose support contains the $j$-th spin and $R_{\alpha}$ belongs to algebra spanned by operators $X_{s}$, whose support contains $j$-th spin. (If the spin does not belong to support of any $s$, then $P_{\alpha}=I$, and similarly for $R$. However, in Kitaev-type models this latter case does not occur). The dissipative generator has the following form

$$
\mathcal{L}=\mathcal{L}_{x}+\mathcal{L}_{z}
$$

where $\mathcal{L}_{x}, \mathcal{L}_{z}$ consist of Davies operators of type $a$ and $b$ respectively. The Davies operators describe the elementary noise processes. In 2D model, they are creation, anihilation and motion of two types of point-like anyons. In $4 \mathrm{D}$ model, excitations are not point-like, and the processes are creation, anihilation and two types of modification of loops (see [8], sec. X, and secs. V, III A of the present paper).

Consider now observables of the form $X_{S}$ and $Z_{T}$, where $S, T$ are some subsets of spins. Let us assume that $X_{S}$ and $Z_{T}$ commute with all $X_{s}$ and $Z_{c}$. Then $X_{S}$ commutes with Davies operators of type $a$ and $Z_{T}$ commutes with Davies operators of type $b$. Therefore from (7) we get that

$$
\mathcal{L}\left(X_{S}\right)=\mathcal{L}_{z}\left(X_{S}\right), \quad \mathcal{L}\left(Z_{T}\right)=\mathcal{L}_{x}\left(Z_{T}\right)
$$

Consider now a modification of the model. Let the Hamiltonian be of the form

$$
H=-\sum_{s} X_{s}
$$

and the coupling with environment be of the form

$$
H_{i n t}=\sum_{j} \sigma_{z}^{j} \otimes \tilde{f}_{j} .
$$

Then dissipative generator for this model consists of Davies operators (34) i.e. it is given just by $\mathcal{L}_{z}$. We obtain 
Proposition 2 Let $X_{s}=\Pi_{j \in s} \sigma_{j}^{x}, Z_{c}=\Pi_{j \in c} \sigma_{j}^{z}$ where the sets $s$ and $c$ are chosen in such way that $X_{s}$ and $Z_{c}$ commute for all $s, c$. Consider $X_{S}$ which commutes with all $X_{s}$ and $Z_{c}$. Then

$$
\mathcal{L}\left(X_{S}\right)=\mathcal{L}^{\prime}\left(X_{S}\right)
$$

where $\mathcal{L}$ is dissipative generator coming from

$H=-\sum_{s} X_{s}-\sum_{c} Z_{c}, \quad H_{i n t}=\sum_{j} \sigma_{j}^{x} \otimes f_{j}+\sum_{j} \sigma_{j}^{z} \otimes \tilde{f}_{j}$.

and $\mathcal{L}^{\prime}$ is dissipative generator coming from

$$
H^{\prime}=-\sum_{s} X_{s}, \quad H_{i n t}^{\prime}=\sum_{j} \sigma_{j}^{z} \otimes \tilde{f}_{j} .
$$

Moreover

$$
\operatorname{Tr}\left(\rho_{\beta} X^{\dagger} \mathcal{L}(X)\right)=\operatorname{Tr}\left(\rho_{\beta}^{\prime} X^{\dagger} \mathcal{L}^{\prime}(X)\right),
$$

where $\rho_{\beta}=\frac{1}{\mathcal{Z}} e^{-\beta H}$ and $\rho_{\beta}^{\prime}=\frac{1}{\mathcal{Z}^{\prime}} e^{-\beta H^{\prime}}$ respectively. Analogous result holds for $Z_{T}$, which commutes with all $X_{s}$ and $Z_{c}$.

Remark. Further in text, $\langle\cdot, \cdot\rangle_{\beta}$ will denote scalar product with the Gibbs state of type $\rho_{\beta}^{\prime}$ (with suitable $H^{\prime}$, depending whether we talk about $X$ or $Z$ ).

\section{A. Observables $X$ and $Z$}

\section{3D Kitaev model}

The Hamiltonian for 3D Kitaev model is given by [8]

$$
H=-\sum_{s} X_{s}-\sum_{c} Z_{c}
$$

where each $s$ denotes set of four plaquettes which share common link, and and each $c$ is six plaquettes forming cube. We will now define a class of observables of interest. To this end we will use observable $X_{C}$ with $C$ being set of parallel plaquettes forming a loop that winds around the torus (there are three homologically inequivalent choices, we will consider a fixed one of them). Such observable is very unstable, hence we may call it "bare qubit observable". One needs to "dress" it with another dichotomic observable which would store the error syndrome. The latter observable will then belong to the abelian algebra spanned by star observables $X_{s}$, hence depending solely on atomic projectors of the algebra which correspond to configurations $\mathcal{K}$ of excited links (stars can be labeled by the links - their centers). Let us call the projectors $P_{\mathcal{K}}$. The needed observable will be thus of the form

$$
F_{x}=\sum_{\mathcal{K}} \lambda_{\mathcal{K}} P_{\mathcal{K}}
$$

where $\lambda_{\mathcal{K}}= \pm 1$. We shall not determine the values of $\lambda_{\mathcal{K}}$ at the moment. They will emerge from our analysis of stability in sec. V and will be then described in sec. VI.

The full "dressed observable" is the product $X_{C} F_{x}$. According to Proposition 2 it evolves according to classical model with Hamiltonian

$$
H_{X}=-\sum_{s} X_{s}
$$

coupled to environment via operators $\sigma_{j}^{z}$. The model is known as $Z_{2}$ gauge Ising model (the Ising variables are in our case eigenvectors of $\sigma_{j}^{x}$ ) [23].

One can define analogous observable $Z_{P} F_{z}$. However in $3 D$ there will be no $\mathrm{X}-\mathrm{Z}$ symmetry. The observable $Z_{P}$ is associated with plane, and atomic projector of algebra spanned by $Z_{c}$ is labeled by configurations of points (i.e. the plaquettes) rather than by loops. Observable $Z_{P} F_{z}$ is evolving according to the model with $H_{Z}=-\sum_{c} Z_{c}$ coupled via $\sigma_{j}^{x}$. It will not be stable (as pointed out in [8]) and most likely, one can prove it by use of techniques worked out in [17].

\section{4D Kitaev model}

In four dimensional model the spins again sit on plaquettes, and the Hamiltonian is similar as in 3D case:

$$
H=-\sum_{s} X_{s}-\sum_{c} Z_{c}
$$

The only difference is that the star $s$ has six plaquettes, because there is six plaquettes common to a single link. Thanks to it there is symmetry: We fix two planes $p_{1}$ and $p_{2}$ on the lattice and on the dual lattice, respectively, obtaining bare qubit observables $X_{p_{1}}$ and $Z_{p_{2}}$. Then candidates for stable observables will be the dressed ones $X_{p_{1}} F_{x}, Z_{p_{2}} F_{z}$. The latter will again evolve separately, and since $4 D$ lattice is self-dual, the evolutions are the same. We arrive at the $4 D Z_{2}$ gauge Ising model.

If we prove that e.g. observable of the form $X_{p_{1}} F_{x}$ is stable, then also similar $Z_{p_{2}} F_{z}$ will be stable too, so that we will obtain stable qubit.

\section{STABILITY CONDITIONS FOR KITAEV MODEL}

\section{A. Bound for decay rate for dressed observables}

The bound (30) applied to generator consisting of Davies generators $(33),(34)$ takes the form

$$
\begin{aligned}
& -\langle A, \mathcal{L}(A)\rangle_{\beta} \leq 2 \hat{h}_{\max } \sum_{j}\left\langle\left[\sigma_{j}^{x}, A\right],\left[\sigma_{j}^{x}, A\right]\right\rangle_{\beta}+ \\
& +\sum_{j}\left\langle\left[\sigma_{j}^{z}, A\right],\left[\sigma_{j}^{z}, A\right]\right\rangle_{\beta} .
\end{aligned}
$$

The quantity $h_{\max }$ given by (27) is a constant independent of the size of the system. This is due to the fact that 
Kitaev models exhibits strong locality property, implying that there is a constant number of frequencies involved in the generator (e.g. just one positive frequency in $2 \mathrm{D}$ model) which are independent of the number of spins $N$.

Since the observables $Z=Z_{P} F_{z}, X=X_{C} F_{x}$ (or analogous ones from $4 \mathrm{D}$ model) commute with Hamilotnian, the bound is applicable. We obtain

$$
\begin{gathered}
-\langle X, \mathcal{L}(X)\rangle_{\beta} \leq 4 \hat{h}_{\max } \sum_{j}\left(1-\left\langle X, \sigma_{j}^{z} X \sigma_{j}^{z}\right\rangle_{\beta}\right) \\
-\langle Z, \mathcal{L}(Z)\rangle_{\beta} \leq 4 \hat{h}_{\max } \sum_{j}\left(1-\left\langle Z, \sigma_{j}^{x} Z \sigma_{j}^{x}\right\rangle_{\beta}\right)
\end{gathered}
$$

where $j$ runs over all spins. We see that the problem of decay of time autocorrelation function has been reduced to the much simpler problem of "one step" autocorrelation function.

\section{B. Gibbs state is concentrated on configurations without long loops}

First we will estimate probability that a configuration has loop of length $l$. We shall use the Peierls argument following Dennis et al. [8] and Griffiths [24]. To this end we first estimate probability that a fixed loop $\lambda$ with length $l$ emerges. Let $\mathcal{C}$ be the set of all configurations which contain loop $\lambda$. The probability is then given by

$$
P(\lambda)=\frac{\sum_{\mathcal{K} \in \mathcal{C}} e^{-\beta E(\mathcal{K})}}{\sum_{\mathcal{K}} e^{-\beta E(\mathcal{K})}}
$$

where in denominator we have sum over all configurations. For any configuration $\mathcal{K}$ containing $\lambda$ we flip spins on a chosen surface whose boundary is $\lambda$, obtaining new configuration $\mathcal{K}^{*}$ which differs from $\mathcal{K}$ only in that the loop $\lambda$ is not present anymore. Hence $E(\mathcal{K})=E\left(\mathcal{K}^{*}\right) e^{-\beta l}$ (or the quantities here are taken to be dimensionless). Thus we write

$$
P(\lambda)=\frac{e^{-\beta l} \sum_{\mathcal{K}^{*} \in \mathcal{C}} e^{-\beta E\left(\mathcal{K}^{*}\right)}}{\sum_{\mathcal{K}} e^{-\beta E(\mathcal{K})}}
$$

Leaving in denominator only configurations $\mathcal{K}^{*}$, we can only decrease it, so that $P(\lambda) \leq e^{-\beta l}$.

Now, the probability $P(l)$ of appearing a configuration which has a loop of length $l$ is bounded by the number of all possible loops of length $l$ times $e^{-\beta l}$. A trivial bound for the number of loops in cube of linear size $L$ in dimension $d$, that start from a fixed node is $2 d(2 d-1)^{l}$. This should be multiplied by the number of nodes, which is proportional to the volume i.e. polynomial in linear size $L$ of the system. Finally, we obtain that

$$
P(l) \leq \operatorname{poly}(L) \mu^{l} e^{-\beta l}=\operatorname{poly}(L) e^{-l(\beta-\ln \mu)}
$$

where $\mu$ is a constant depending only on $d$. Thus below certain critical temperature $T_{\text {crit }}$ we have

$$
P(l) \leq \operatorname{poly}(L) e^{-\delta l}
$$

where $\delta=\beta-\ln \mu$ is positive and does not depend on the size of the system. We then evaluate probability of appearing a configuration that has a loop greater than $L^{\prime}$

$$
P\left(l \geq L^{\prime}\right) \leq \operatorname{poly}(L) \sum_{l=L^{\prime}}^{\infty} e^{-\delta l}=\operatorname{poly}(L) e^{-\delta L^{\prime}} \frac{1}{1-e^{-\delta}}
$$

Thus we see that below $T_{\text {crit }}$ the probability of obtaining e.g. a loop of length $L / 8$ or greater is exponentially decaying in $L$.

\section{Stability of Kitaev 4D model}

In next section we shall prove that for configurations having only loops shorter than $L^{\prime}=L / 8$ a single flip does not change observables $X$ and $Z$ for Kitaev $4 \mathrm{D}$ model. This implies that

$$
\sum_{j}\left(1-\left\langle Z, \sigma_{j}^{x} Z \sigma_{j}^{x}\right\rangle_{\beta}\right) \leq \sum_{j} 2 P\left(l \geq L^{\prime}\right)
$$

so that

$$
-\langle Z, \mathcal{L}(Z)\rangle \leq \operatorname{poly}(L) e^{-\delta^{\prime} L}
$$

where $\delta^{\prime}=\delta / 8$ is a constant that is positive below some critical temperature. The same happens for observable $X$, hence due to proposition 1 the decay time of fidelity is exponentially long in size of the system.

\section{STABILITY OF TOPOLOGICAL OBSERVABLES}

In previous section we have shown that below certain critical temperature $T_{\text {crit }}$ the Gibbs state is concentrated on configurations with short loops. Thus if on such configurations an observable does not change under single spin flip, it is stable within the classical model. If in addition it is of the special form $X_{C} F_{x}$, then it is also stable within the quantum model (see section $\mathrm{VC}$ ).

In this section we shall build such observable. To this end we shall first define homology classes of spin configurations corresponding to configurations of loops with short loops only. We will then show that, as expected, single spin flip does not change those homology classes. This implies that any observable which depends solely on the homology classes does not change under single spin flips (for configurations containing only short loops). This result holds for torus of any dimension. Subsequently we shall show, that some observables of the form $X_{C} F_{x}$ share this property. 


\section{A. Observables depending only on homology classes}

Let us introduce some notation. By $S$ we will denote configuration of spins on the lattice (in the form of configurations of bits whose values encode spin orientation). Given two spin configurations $S_{1}$ and $S_{2}$, we can add them to obtain new configuration $S$. We denote it by $S=S_{1} \oplus S_{2}$, and the addition is bit-wise, modulo 2 . I.e. if at given site the spins are the same, resulting spin is down, if they are different, resulting spin is up. We denote by $S_{0}$ configuration of all spins down.

By $\mathcal{K}$ we will denote set of excited links. A link is excited, if the parity of spins on adjacent plaquettes is odd (in 3D a link has four such plaquettes, and in 4D six ones). One finds that $\mathcal{K}$ is sum of disjoint loops $l_{j}$ (the loops can have self crossing at nodes):

Lemma 1 For given configuration $\mathcal{K}$ consider a connected set of links. It is sum of closed loops, which visit each link and each node at most one time. Equivalently, it is a closed walk, which visit one link only at most once.

Proof. The proof is by induction.

We will call such connected sets "loops". We will say that a loop is short, when its length is no greater than $c L$, where $c$ is a fixed constant, which we can take e.g. $1 / 8$.

Any spin configuration $S$ defines link configuration $\mathcal{K}$. We will then write $S(\mathcal{K})$. Of course for given $\mathcal{K}$ there are many spin configurations leading to them. Sometimes for given $S$ the corresponding $\mathcal{K}$ will be denoted by $\partial S$ and called boundary of $S$.

Definition 1 By continuous deformation of spin configuration we mean operation, which can be composed of the following elementary operations: flipping spins on all plaquettes belonging to an elementary d-dimensional cubes.

Remark 1. Continuous deformation does not change the configuration of links. For 3D easy to see: indeed, flipping spins on faces of cube, change at the same time spins on two plaquettes adjacent to a link from the cube.

Definition 2 We say that $S_{1}$ and $S_{2}$ with empty boundary are homologically equivalent if they can be transformed into one another by continuous deformation. S is called homologically trivial, if it can be continuously transformed into $S_{0}$.

Definition 3 We say that $S_{1}$ and $S_{2}$ which have the same boundary are homologically equivalent and denote it by $S_{1} \sim S_{2}$, if $S_{1} \oplus S_{2}$ is homolgically trivial

Definition 4 ("Shortest configuration") Consider given $\mathcal{K}=\bigcup_{j} l_{j}$. For each loop $l_{j}$ fix a shortest surface whose boundary is $l_{j}$. Consider then $S^{*}\left(l_{j}\right)$ which has spins up on this surface and all other spins down. The configuration $S^{*}=\oplus_{j} S^{*}\left(l_{j}\right)$ will be called shortest configuration for $\mathcal{L}$.
Fact 1 All shortest configurations $S^{*}$ for given $\mathcal{K}$ are homologically equivalent, provided $\mathcal{K}$ contains only short loops.

Proof. Take two different shortest configurations. We have

$$
S_{1}^{*} \oplus S_{2}^{*}=\oplus_{j}\left[S_{1}^{*}\left(l_{j}\right) \oplus S_{2}^{*}\left(l_{j}\right)\right]
$$

However, each configuration $S_{1}^{*}\left(l_{j}\right) \oplus S_{2}^{*}\left(l_{j}\right)$ is trivial. Indeed, since loop $l_{j}$ is short then $\left|S_{1}^{*}\left(l_{j}\right)\right|$ and $\left|S_{2}^{*}\left(l_{j}\right)\right|$ are small, and cannot form homologically nontrivial surface.

Definition 5 For $\mathcal{K}$ containing only short loops, with any $S$ leading to $\mathcal{K}$ we can associate the homology class of $S \oplus S^{*}(\mathcal{K})$. Denote it by $h(S)$.

Remark 2. For fixed $\mathcal{K}$ obviously $S_{1} \sim S_{2}$ iff $h\left(S_{1}\right)=$ $h\left(S_{2}\right)$. Thus the above definition allows to ascribe labels to homology classes of spin configuration, by relating to distinguished class i.e. the class of $S^{*}$. But the homology classes are now defined for any $\mathcal{K}$. Thus we will be able to ask later, whether a spin flip (which of course changes $\mathcal{K})$ can preserve homology class. For any $\mathcal{K}$ there are eight homology classes in $3 \mathrm{D}$ case, associated with three possible ways of winding around torus. In $4 D$ there is 16 classes.

We have obvious fact:

Fact 2 We have $S_{1} \oplus S_{2}=\sigma_{j}\left(S_{1}\right) \oplus \sigma_{j}\left(S_{2}\right)$, where $\sigma_{j}$ flips $j$-th spin.

Now we will show that for short loops, single spin flip does not change homology class of $S$. To this end we first prove the following lemma

Lemma 2 For $\mathcal{K}$ containing only short loops we have

$$
\sigma_{i}\left(S^{*}(\mathcal{K})\right) \sim S^{*}\left(\sigma_{i}(\mathcal{K})\right) .
$$

Here $\sigma_{i}(\mathcal{K})$ is understood as the configuration of loops arising from configuration $\mathcal{K}$ by applying $\sigma_{i}$

Proof. Divide $\mathcal{K}$ into two sets: $\mathcal{K}_{1}$ consisting of loops that contain some links from $i$-th plaquette, and $\mathcal{K}_{2}$ which does not contain links from this plaquette. Then $\sigma_{i}(\mathcal{K})=\sigma_{i}(\mathcal{K}) \cup \mathcal{K}_{1}$ hence

$$
\sigma_{i}\left(S^{*}(\mathcal{K})\right)=\sigma_{i}\left(S^{*}\left(\mathcal{K}_{1}\right)\right) \oplus S^{*}\left(\mathcal{K}_{2}\right)
$$

and

$$
S^{*}\left(\sigma_{i}(\mathcal{K})\right)=S^{*}\left(\sigma_{i}\left(\mathcal{K}_{1}\right)\right) \oplus S^{*}\left(\mathcal{K}_{2}\right) .
$$

Thus only $\mathcal{K}_{1}$ is in the game:

$$
\sigma_{i}\left(S^{*}(\mathcal{K})\right) \oplus S^{*}\left(\sigma_{i}(\mathcal{K})\right)=\sigma_{i}\left(S^{*}\left(\mathcal{K}_{1}\right)\right) \oplus S^{*}\left(\sigma_{i}\left(\mathcal{K}_{1}\right)\right)
$$

and therefore we have to show that right-hand-side of the above formula is homologically trivial. Indeed, the set $\mathcal{K}_{1}$ contains at most two loops independently of dimension. Now, since loops are short, both $\sigma_{i}\left(S^{*}\left(\mathcal{K}_{1}\right)\right)$ and $S^{*}\left(\sigma_{i}\left(\mathcal{K}_{1}\right)\right)$ are small, and added together must give a trivial surface.

Now we are in position to prove the main result of this section 
Proposition 3 Consider configuration of spins $S$ for which $\mathcal{K}$ has short loops only. Then single spin flip does not change the homology class of $S$. More explicitly, we have

$$
\sigma_{i}(S) \oplus S^{*}\left(\sigma_{i}(\mathcal{K})\right) \sim S \oplus S^{*}(\mathcal{K})
$$

Proof. By lemma 2 we have

$$
\sigma_{i}\left(S^{*}(\mathcal{K})\right) \sim S^{*}\left(\sigma_{i}(\mathcal{K})\right)
$$

By fact 2 we have

$$
\sigma_{i}(S) \oplus \sigma_{i}\left(S^{*}(\mathcal{K})=S \oplus S^{*}(\mathcal{K})\right.
$$

Combining the above two equations, we obtain the claim.」

Thus any observable $\mathcal{T}$ which for configurations $\mathcal{K}$ containing only short loops depends only on homology class, i.e.

$$
\mathcal{T}(S)=\mathcal{T}(h)
$$

is dynamically stable within the model of Proposition 2 below some critical temperature.

\section{B. Construction of stable topological observables}

Our bare observable will be $X_{T}=\Pi_{j \in T} \sigma_{x}^{j}$ where $T$ is chosen in such a way that $X_{T}$ is invariant under flipping spins on plaquettes from any cube (i.e. it is invariant under continuous transformations). Examples of such observables exists, as will be shown later in next subsection. We will show that one can find dichotomic observable $F_{x}$ which will depend on given configuration $S$ only through $\mathcal{K}$, such that the dressed observable $X_{T} F_{x}$ depends only on homology of $S$ (for short loops) i.e. it is stable within the classical model.

We begin with the following lemma

Lemma 3 The observable $X_{T}$ which is invariant under flipping spins on plaquettes of any cube is constant on homology classes for any fixed link configuration $\mathcal{K}$ containing only short loops (cf. definition 5).

Remark. Note that this does not mean that $X_{T}$ is stable. Indeed, for any fixed link configuration, it is constant on the whole homology classes. However if the link configuration changes, it may change sign on the same homology class. The stable observable described in previous subsection has the same value on a given homology class independently of link configurations, provided there are only short loops.

Proof. Consider arbitrary spin configurations $S$ and $S^{\prime}$ whose boundary is $\mathcal{K}$, and which are in the same class of homology, i.e. $S_{1} \equiv S \oplus S^{*}$ is homologically equivalent to $S_{2} \equiv S^{\prime} \oplus S^{*}$. Therefore $S_{1}$ can be transformed into $S_{2}$ by flipping spins on a set of elementary cubes. This does not change the sign of $X_{T}$, so that $X_{T}$ has the same sign on $S_{1}$ and $S_{2}$. Thus it has the same sign also on $S$ and $S^{\prime}$.

Now we are in position to build stable observable. Now let us assume that

$$
X_{T}\left(S_{1}\right) X_{T}\left(S_{2}\right)=X_{T}\left(S_{1} \oplus S_{2}\right) .
$$

We stress here that this assumption is easily seen to hold for particular observables considered in next subsection. (One can actually show, that it is true in general for observables satisfying assumptions of the above lemma). Using this we can write

$$
X_{T}(S)=X_{T}\left(S \oplus S^{*} \oplus S^{*}\right)=X_{T}\left(S \oplus S^{*}\right) X_{T}\left(S^{*}\right)
$$

Since homology class of $S^{*}$ is always the same for short loops (independently on possible amiguity of $S^{*}$ for given loop) then $X_{T}\left(S^{*}\right)$ depends only on the loops configuration: $X_{T}\left(S^{*}\right)=X^{\prime \prime}(\mathcal{K})$, so that $X_{T}(S)=X_{T}(S \oplus$ $\left.S^{*}\right) X^{\prime \prime}(\mathcal{K})$. Now, since for fixed loops configuration $X_{T}$ depends only on homology class and the loops configuration for $S \oplus S^{*}$ is always null (as $S \oplus S^{*}$ does not have a boundary), we get that $X_{T}\left(S \oplus S^{*}\right)$ depends only on homology class of $S \oplus S^{*}$. Therefore, according to definition 5, it depends only on homology $h$ of $S$. Hence $X_{T}\left(S \oplus S^{*}\right)=X^{\prime}(h)$ and we have

$$
X_{T}(S)=X^{\prime}(h) X^{\prime \prime}(\mathcal{K}) .
$$

Then the following observable

$$
\mathcal{T}(S)=X_{T}(S) X^{\prime \prime}(\mathcal{K})
$$

depends only on $h$. The above observable is defined unambiguously only for spin configurations leading to short loops configurations. This is because $X^{\prime \prime}$ is only well defined only on short loops configurations. We then extend the definition of $\mathcal{T}$ to all spin configurations, by letting $X^{\prime \prime}(\mathcal{K})=1$ for all other loops configurations. Thus we shall take $F_{x}=X^{\prime \prime}$ and obtain that $X_{T} F_{x}$ depends only on homology of spin configuration, hence is stable within classical model.

\section{Observable stable within quantum model}

The observable constructed in the previous subsection is stable within classical model, because it depends only on homology class. However, we know that only special observables from the classical model evolve in the same way in quantum model. E.g. the observables of the form $X_{T} F_{x}$, where $F_{x}$ is from algebra generated by star operators $X_{s}$,a and $X_{T}$ commutes with $Z_{c}$. Here we shall focus on construction of $X_{T}$ since it determines $F_{x}$ via considerations of the previous section.

Now, let us note that the first condition means simply that $F_{x}$ depends only on loops. The second condition means that $X_{T}$ does not change under flips on all plaquettes of an elementary cube. Thus the observable (68) is of the above form, hence it evolves in the same way 
both in quantum and classical model, hence it is stable also within quantum model.

The last thing is to assure that the observable $\mathcal{T}$ is nontrivial, i.e it is not identity. To this end we have choose the set $T$ in a special way, such that on spin configurations without boundary, $X_{T}$ can take different sign.

For $3 D$ it will be nontrivial loop in dual lattice, i.e. straight line consisting of parallel plaquettes. The fact that it is loop in dual lattice, implies that it $X_{T}$ is invariant under continuous transformations. Since it is nontrivial, then $X_{T}$ have value -1 for spin configuration consisting of plane of flipped spins perpendicular to $T$, while it takes value 1 on homologically trivial spin configurations. Since there are three possible choices of inequivalent nontrivial loops, we can construct three independent observables.

In $4 D$ we take $T$ to be plane in dual lattice, i.e. the value observable $X_{T}$ is defined as a product of values of all plaquettes belonging to the plane $T$. Again, $X_{T}$ does not change under flipping spins on cube because arbitrary cube has exactly two plaquettes in common with such a plane. For this reason it will be also 1 on homologically trivial spin configurations. However it will take value -1 on the configuration consisting of flipped spins on a plane $T^{\prime}$ whose intersection with $T$ is a single plaquette. Note that since there are six homologically nontrivial planes, we can construct six independent observables of this sort.

Now, since the torus in $4 D$ is selfdual, we can consider dual observable i.e. $\mathcal{T}_{z}=Z_{T}^{\prime} F_{z}$, and $F_{z}$ depends only on configuration of three dimensional cubes (such cubes are dual to link). Since $F_{z}$ and $F_{x}$ commute, and planes $T$ and $T^{\prime}$ intersect only in a single plaquette, we obtain that $\mathcal{T}_{z}$ and $\mathcal{T}_{x}$ anticommute, so that they form a qubit.

\section{POLYNOMIAL ALGORITHM FOR MEASURING THE TOPOLOGICAL OBSERVABLES}

The observables are symmetric, so it is enough to show algorithm for one of them, say $\mathcal{T}_{z}$. The algorithm is the following.

1. Measure all spins.

2. Multiply outcomes on a fixed plane in dual lattice, this gives "raw value" of the observable.

3. Identify the loops.

4. For "short" loops we identify associated surfaces (the ones homologically equivalent to shortest ones).

5. If an odd number of surfaces crosses a fixed plane in dual lattice, multiply the "raw value" with -1 .

The step 2 corresponds to measuring the bare observable $X_{T}$, while the steps 3-5 define observable $F_{x}$. The multiplication in last step produces the stable, dressed
A)
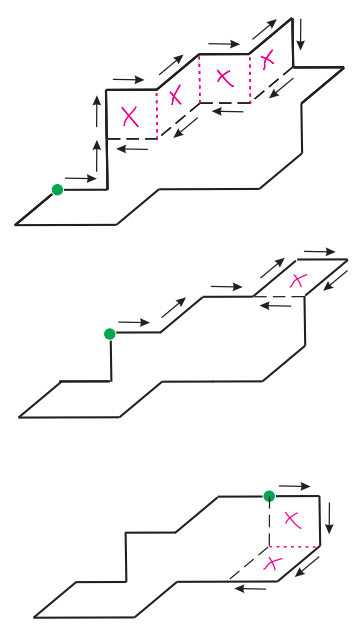

B)
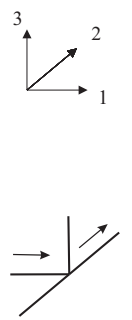

FIG. 1: Efficient algorithm for determining surface closing the loop.

observable $\mathcal{T}_{x}=X_{T} F_{x}$. The only nontrivial problem here is to argue that the step 4 is polynomial. It is actually enough to show that for a fixed loop, one can find efficiently a surface which is contained in the smallest cube containing the loop.

Moreover, it is enough to find a protocol which in efficient way allows to find spins which, if flipped, reduce length of the loop by some amount (in our protocol, it will be reduced by two).

The protocol is the following. We first choose a Cartesian frame. We start with a link of the loop, and move along the loop. If there is ambiguity (the loop crosses itself) the priority is set by the chosen frame: if only we can we go in positive direction of the axis with the smallest number. If not, then we go in negative direction of the axis with the smallest number. The same rule governs choice of the starting link.

The walk is stopped, if we are forced at some point to go in opposite direction to any of the previous steps (see figure).

When the walk is stopped, the link at which we stopped and the last "opposite" link, determine uniquely the set of plaquettes. This is because all the links of the walk lying between two "opposite links" are perpendicular to them. Now, after flipping spins on the set of plaquettes, the two opposite links are removed from the curve. Note that this flipping may further diminish the length of loop, if by a chance, the chose plaquettes have some other links common with the loop. It may also divide the loop into smaller ones, however their joint length is not longer than $l-2$.

\section{CONCLUDING REMARKS}

We have shown that within Markovian weak coupling approximation, there exist a stable quantum subsystem in four dimensional Kitaev model of [8]. While the qubit 
is indeed stable, there are several other drawbacks, which makes the question of existence of self correcting quantum memory still open. Minimal requirement for good quantum memory is that it should allow to encode arbitrary state of qubit (encoding), then to store it for long time (storage) and finally perform a measurement in arbitrary basis (readout). It would be also good if the measurement is repeatable. The present result shows that storage is possible, but does not touch the problem of preparation and measurement. Actually, the algorithm for measuring topological observables is highly destructive, hence non-repeatable. The encoding and read-out one usually performs by preparing qubit in a standard state, and also measure standard observable, the rest being done by gates. Also repeatability can be then assured, if one can perform c-not gates on the protected qubits. However the problem with the Kitaev's model is that it does not support universal computation. A possible solution of this problem is to use the version of topological quantum memory developed by Bombin and Delgado [25] which supports universal computation (we shall present the dynamical analysis of these models elsewhere). How- ever, still there is a separate problem of preparation of the qubit in standard state.

Finally, let us mention, that the present result has separate implications in statistical physics. In the standard approach to large quantum systems the metastable states of encoded qubits like those found for Kitaev models disappear in the thermodynamic limit merging into a classical simplex of equilibrium (KMS) states [11]. On the other hand they carry an interesting topological structure which might be physically relevant. In this context it is interesting to ask for a new description of infinite system, which would take into account such new metastable states. In particular, phase transitions which lead to such curious states require further investigations.

\section{Acknowledgements}

We are grateful to John Preskill for drawing our attention to results of Ref. [8] on $4 \mathrm{D}$ model and for numerous discussions. We thank Mark Fannes for stimulating discussions. M.H. would like also to thank Hector Bombin and Miguel Martin-Delgado for helpful discussions. This work is supported by the EU Project QAP-IST contract 015848 and EC IP SCALA.
[1] M. A. Nielsen and I. L. Chuang, Quantum Computation and Quantum Information (Cambridge University Press,Cambridge, 2000).

[2] D. Aharonov and M. Ben-Or (1996), quant-ph/9611025.

[3] E. Knill, R. Laflamme, and W. H. Zurek, Science 279, 342 (1998).

[4] R. Alicki, M. Horodecki, P. Horodecki, and R. Horodecki, Phys. Rev. A 65, 062101 (2002), quant-ph/0105115.

[5] B. M. Terhal and G. Burkard, Phys. Rev. A 71, 012336 (2005), quant-ph/0402104.

[6] D. Aharonov, A. Kitaev, and J. Preskill, Phys. Rev. Lett. 96, 050504 (2006), quant-ph/0510231.

[7] R. Alicki (2004), quant-ph/0402139.

[8] E. Dennis, A. Kitaev, A. Landahl, and J. Preskill, J. Math. Phys. 43, 4452 (2002), quant-ph/0110143.

[9] A. Y. Kitaev, Annals Phys. 303, 2 (2003), quant$\mathrm{ph} / 9707021$.

[10] H. Bombin and M. A. Martin-Delgado, Physical Review B 75, 075103 (2007), cond-mat/0607736.

[11] R. Alicki and M. Horodecki (2006), quant-ph/0603260.

[12] R. Alicki, M. Fannes, and M. Horodecki, 40, 6451 (2007),
arXiv:quant-ph/0702102.

[13] Z. Nussinov and G. Ortiz (2007), cond-mat/0702377.

[14] Z. Nussinov and G. Ortiz (2007), arXiv:0709.2717.

[15] A. Kay (2008), arXiv:0807.0287.

[16] S. Iblisdir, D. Perez-Garcia, M. Aguado, and J. Pachos (2007), arXive:0806.1853.

[17] R. Alicki, M. Fannes, and M. Horodecki (2008), arXiv:0810.4584.

[18] R. Alicki and L. Lendi, Quantum dynamical semigroups and applications, II edition (Springer, 2008).

[19] H. Spohn, Lett. Math. Phys. 2, 33 (1977).

[20] A. Frigerio, Comm. Math. Phys. 63, 269 (2007).

[21] H. Hofmann, Phys. Rev. Lett. 94, 160504 (2005), quant$\mathrm{ph} / 0411011$.

[22] M. Horodecki, P. Horodecki, and R. Horodecki, Phys. Rev. A 60, 1888 (1999), quant-ph/9807091.

[23] J. B. Kogut, Rep. Math. Phys. 51, 659 (1979).

[24] R. Griffiths, Phys. Rev. A 136, 437 (1964).

[25] H. Bombin and M. Martin-Delgado, Phys. Rev. Lett. 98, 160502 (2007), quant-ph/0610024. 